\title{
Sciendo
}

Transport and Telecommunication, 2022, volume 23, no. 1, 62-72

Transport and Telecommunication Institute, Lomonosova 1, Riga, LV-1019, Latvia

DOI 10.2478/ttj-2022-0006

\section{A NEW METHOD TO BOOST VOIP PERFORMANCE OVER IPV6 NETWORKS}

\author{
Mosleh M. Abualhaj ${ }^{1}$, Sumaya N. Al-Khatib ${ }^{2}$ \\ 1,2 Al-Ahliyya Amman University, Faculty of Information Technology \\ Amman, Jordan, Zip-Code (Postal Address):19328 \\ Im.abualhaj@ammanu.edu.jo
}

The demands on virtual communication have increased noticeably during the COVID-19 pandemic lockdown. As an essential part of virtual communication, VoIP should be promoted to achieve the desired level of performance. One critical area investigated in VoIP is the bandwidth utilization (BWU) of the VoIP over IPv6 networks. Enhancing BWU will impact call capacity and quality; it increases call capacity and boosts call quality. Unfortunately, a considerable amount of bandwidth is wasted when running VoIP over IPv6 networks. This is due to the large size of the IPv6 header and the small size of the speech frame. This paper proposes a new method to handle the inefficient BWU when running VoIP over IPv6 networks. The proposed method combines multiplexing multiple VoIP packets in one IPv6 header and using the superfluous fields to carry a portion of the speech frame. Therefore, the proposed is called packet multiplexing and carrier fields (PMCF). Investigation of the PCMF method has been done using four metrics to measure the promotion in BWU, namely calls capacity, header size, bandwidth saving, and speech frame shortening metrics. With the four metrics, the PMCF method has outperformed the comparable methods. For instance, the call capacity has been promoted by up to $269 \%$ compared to the typical IPv6 method in the tested scenarios. Therefore, the PMCF method is a feasible solution to facilitate the BWU of VoIP when running VoIP over IPv6 networks.

Keywords: VoIP, bandwidth utilization, IPv6, VoIP performance

\section{Introduction}

At the beginning of 2020, the demand for virtual communications has increased dramatically and rapidly due to the COVID-19 pandemic lockdown. People couldn't meet but needed to communicate for various purposes such as work, education, and even medication (Raycheva et al., 2020; Garro_Abarca et al., 2021). The real-time voice and video over IP have served these purposes to a great degree. As for VoIP (voice over IP), the forecasting for 2021 is around 3 billion subscribers (Hoffman, 2019; Abualhaj et al., 2020a). Therefore, VoIP networks should be enhanced to carry out the calls thoroughly. One of the metrics that should be investigated to enhance the performance of the VoIP networks is bandwidth utilization (BWU). Improving BWU is directly proportional to the call's capacity and quality; it increases call capacity and boosts the call quality (Abualhaj et al., 2020a).

The nature of VoIP traffic is highly depressing the BWU of VoIP networks because the VoIP packet header size is immense compared to the VoIP packet payload. In many cases, the VoIP packet header is larger than the VoIP packet payload. On the one hand, the VoIP packet payload is constructed from one or more digital speech samples produced by a codec. Codecs are making very small speech samples, typically from 10 to 30 bytes. Increasing the speech sample size will increase the latency of the VoIP, which is not acceptable for the call quality. Therefore, the speech sample should be small and thus the VoIP packet payload. For instance, the G.729 codec produces a 10-byte speech sample as a packet payload. Table 1 shows the typical length of the speech sample of the most common codecs (Tomoskozi et al., 2016; Abualhaj et al., 2021a; Gupta et al., 2018).

On the other hand, the VoIP packet header is constructed from three protocols, excluding the layer 2 protocol, namely RTP, UDP, and IPv4/IPv6. In IPv4, the header's length (RTP/UDP/IPv4) is 40-byte, which consumes around $80 \%$ of the total bandwidth with the G.729 codec. The consumed bandwidth is even more in the case of IPv6 protocol. The RTP/UDP/IPv6 header length is 60-byte, consuming 85.7\% of the total bandwidth with the same G.729 codec (Abualhaj et al., 2021a; Narayan, 2010). Table 2 shows the consumed BW when using the IPv6 protocol with different codecs. As we can see, the packet's header consumed an excessive amount of bandwidth, especially when using IPv6 protocol, and depress the quality of the calls. Several researchers have investigated this issue. In this study, further investigation of this issue will be introduced, and a new technique will be designed to make this issue less severe. 
The remaining part of the paper has the following structure: the related works in Section 2 covers some of the main multiplexing methods. The proposed method to handle the BWU issue of VoIP is presented in Section 3. Section 4 investigates the proposed method in comparison to the other method in terms of BWU. Section 5 presents the finding and conclusion of the paper.

Table 1. Common speech codecs

\begin{tabular}{|l|l|l|}
\hline Codec & Sample Size (byte) & Mean Opinion Score (MOS) \\
\hline G.729 & 10 & 4.1 \\
\hline G.728 & 10 & 3.61 \\
\hline G.723.1 & 24 & 3.9 \\
\hline G.726 & 20 & 3.85 \\
\hline
\end{tabular}

Table 2. Consumed BW by packet header

\begin{tabular}{|l|l|l|l|l|}
\hline Codec & Protocol & Common Payload size & Packet size & Header Consumed BW \\
\hline \multirow{2}{*}{ G.729 } & IPv4 & 10 & 50 & $80 \%$ \\
\cline { 2 - 5 } & IPv6 & 10 & 70 & $85.7 \%$ \\
\hline \multirow{2}{*}{ G.728 } & IPv4 & 10 & 50 & $80 \%$ \\
\cline { 2 - 5 } & IPv6 & 10 & 70 & $85.7 \%$ \\
\hline \multirow{2}{*}{ G.723.1 } & IPv4 & 24 & 64 & $62.5 \%$ \\
\cline { 2 - 5 } & IPv6 & 24 & 64 & $714 \%$ \\
\hline \multirow{2}{*}{ G.726 } & IPv4 & 20 & 80 & $66 \%$ \\
\cline { 2 - 5 } & IPv6 & 20 & & $75 \%$ \\
\hline
\end{tabular}

\section{Related Works}

BWU is a critical issue in the VoIP sector because it affects both call capacity and call quality. Many types of research with different approaches have been suggested to alleviate the severity of poor BWU. One of the top approaches is packet multiplexing, which proposing piggybacking many small VoIP packets in one IP header (RTP/UDP/IP) (Abualhaj el at., 2020b; Seytnazarov and Kim, 2017; Sze et al., 2002; Casner and Jacobson, 1999). For elaboration, assume the first packet is transmitted to the destination $\mathrm{D}$. Then, theoretically, all packets transmitted to destination $\mathrm{D}$ through the same route will be piggybacked in the header of the first packet. In general, the packets are piggybacked up to a certain length, period, or other critical VoIP performance metric. Figure 1 elaborates the packets multiplexing approach. In the figure (Figure 1), the NPkt refers to the ordinary VoIP packet, the packet before the multiplexing process, and without any modification. In the same figure (Figure 1), the MPkt refers to the multiplexed VoIP packet, resulting from piggybacking several NPkt packets in one header.

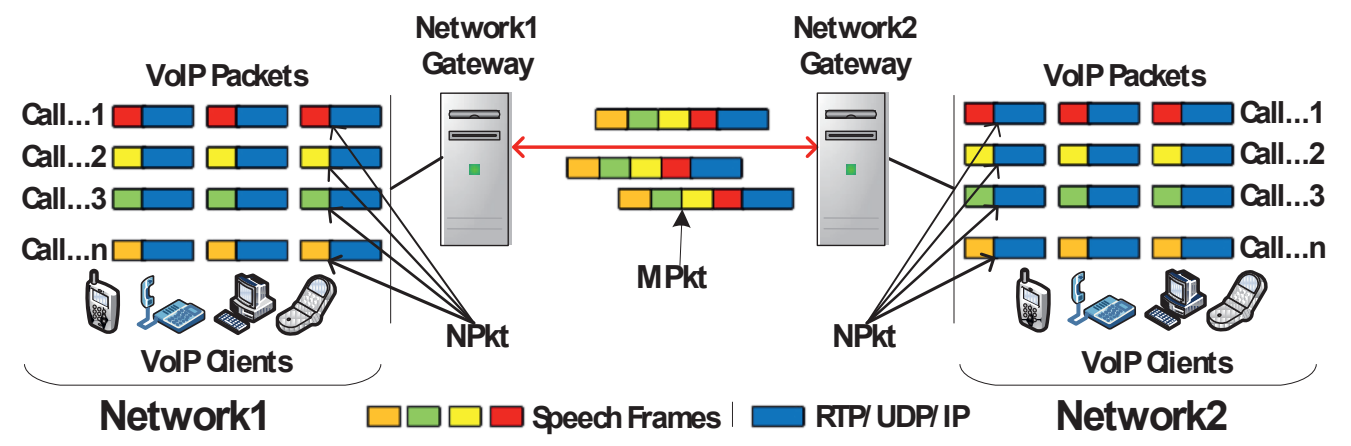

Figure 1. Packet multiplexing approach

Many methods were designed under the multiplexing approach. One of the first methods was developed in Nokia Research Center by Subbiah et al. (Subbiah, 1999). Speech payload from different NPkt packets is piggybacked into one MPkt packet. The multiplexing occurs at the application layer of the OSI stack, in which multiple speech payloads are piggybacked into one RTP/UDP/IP header. A 2-byte header is placed at the beginning of every speech payload to separate the piggybacked speech payloads. The proposed method is designed to work on the VoIP gateways that connect the PSTN/PBX (Public Switched Telephone Network/Private Branch Exchange) systems and cellular access networks. The 
investigation result showed that the BWU of VoIP networks has promoted up to $80 \%$. Besides, the overcrowding in intermediary network devices and processing latency in MPLS (Multiprotocol Label Switching), IPsec, and diff-serv networks are alleviated.

More recently, Abu-Alhaj et al. proposed a multiplexing method called Delta-Multiplexing (Abualhaj et al., 2010). Similar to the previous method, the multiplexing process in Delta-Multiplexing occurs at the transport layer of the OSI stack, in which multiple speech payloads and an RTP header for each payload are piggybacked into one UDP/IP header. Besides the multiplexing, the Delta-Multiplexing method applies a delta algorithm at the speech payload. That is, the delta algorithm finds the difference between the successive packets' speech payload. Only the difference between the speech payload is sent rather than sending the full-length speech payload. The delta algorithm performs the opposite operations at the receiver side to construct the original full-length speech payload. A 2-byte header is placed at the beginning of every mini-packets (RTP header + speech payload) within the MPkt packet to reconstruct the NPkt packets at the receiver side. The multiplexing process and delta algorithm have promoted BWU of VoIP networks between $68 \%$ and $72 \%$.

One of the best multiplexing methods was invented and patented by Roay under the auspices of Cisco Technology Inc. (Roay, 2013). The multiplexing occurs at the network layer of the OSI stack, in which multiple mini-packets (RTP/UDP header + speech payload) are piggybacked into one IP header (IPv4 or IPv6). Only the first mini-packet within the MPkt packet has a flag that indicates that this is an MPkt packet. This flag is used by the receiver gateway to process the incoming packet as an NPkt packet or an MPkt packet. The ability of the receiving gateway to process the MPkt packet is negotiated through call establishment using either SIP or H.323 signaling protocols. If the receiving gateway cannot process the MPkt packet, all the packets to that gateway must be NPkt packets. The MPkt packet does not wait for the NPkt packet to be available but rather piggybacked only the NPkt packets in the queue. This is to avoid imposing an additional delay that may impact the call quality. If the MPkt packet reaches the MTU, it is sent to the destination, even if more NPkt packets are in the queue. The saved bandwidth is 20-byte in IPv4 or 40-byte in IPv6 for every NPkt packet within the MPkt packet.

All the previous works are proposing a multiplexing method to promote the BWU. The method proposed by Roay is designed to be compatible with the current environment since it integrates the RTP/UDP header in every mini-packet. In addition, unlike the other methods, it does not impose any extra delay that impacts the quality of the calls. Moreover, Roay method is the only, up to my knowledge, that discussed and considered IPv6 networks. This makes it a suitable multiplexing method for the delaysensitive VoIP applications over IPv6 networks. Though, adding the RTP/UDP header to every minipacket still degrades the BWU severely. Accordingly, this paper adopts the same method proposed by Roay. Still, it uses the superfluous fields in the headers to carry the speech payload of a packet. This will keep the features of the Roay method and promotes the BWU. The proposed is called packet multiplexing and carrier fields (PMCF). The PMCF method will be designed to work over IPv6 networks.

\section{PMCF Method}

The PMCF method is destined to promote the BWU when running VoIP over IPv6 networks. Multiplexing more VoIP packets in one MPkt packet will help to maximize the BWU. Therefore, the PMCF method should be deployed in a network location where sufficient VoIP packets are passed. This location might be the exit point of a LAN with many VoIP clients or even the exit point of several LANs, each of them with many VoIP clients. In Figure 1, the Netwrok1 gateway is the location at which the PMCF method could be implemented. The PMCF method executes two primary mechanisms: the PMCF sender side (PMCFS) and PMCF receiver side (PMCFR). The PMCFS and PMCFR mechanisms will be explained in Subsection 3.2 and 3.3, respectively.

\subsection{Superfluous Fields}

The VoIP packet consists of three headers: RTP, UDP, and IPv4/IPv6 (IPv6 is the focus of this work). These 60-byte RTP/UDP/IPv6 headers contain much information that serves diverse applications other than VoIP. Only part of this information is necessary to convey the speech payload to the intended destination. The other part of the RTP/UDP/IPv6 headers information is superfluous for VoIP applications and used by other applications. In VoIP applications, this information is conveyed with every packet meaninglessly, creating an unfavorable reduction of BWU (Abualhaj et al., 2021b; Tomsho, 2019; Hartpence, 2013; Perkins, 2003; Gao et al., 2019). The PMCF method will turn the headers' fields that carry this information to carrier fields for the speech payload itself. This way, the speech payload is shortened, and the BWU is promoted. 
The first header of the VoIP packet is the RTP, which precisely conveys the real-time data in all scenarios. Though part of the information in RTP is not used to represent the speech payload in specific scenarios, it will be used in other scenarios. Therefore, the information in the RTP header should be stay as is to make sure that the PMCF method works in all VoIP scenarios (Hartpence, 2013; Perkins, 2003). The second header of the VoIP packet is the UDP, which is used with various applications other than realtime applications. Some of the information in the UDP header is optional and disabled (set to zeros) for many applications, including the VoIP applications, namely the Checksum and the Source Port. Therefore, the fields that carry the Checksum and the Source Port information will be used by the PMCF method as carrier fields for the speech payload (Tomsho 2019; Eggert et al., 2017; Khan et al., 2016; Stein et al., 2020). The third header of the VoIP packet is the IPv6, which is used with all applications in the IP-based networks. VoIP applications do not need the Source IPv6 address information because VoIP applications are not "request/response" applications. However, there is only one IPv6 header with one Source IPv6 address field to the entire MPkt. Therefore, it is not worth using the Source IPv6 address field as a carrier field to the speech payload. In addition, there are several NPkt from different sources within the MPkt. Hence, which of the NPkt's speech payload will be carried by the Source IPv6 address field. This will complicate the process with no real benefit.

In a nutshell, the Checksum and the Source Port fields in the UDP header will be used by the PMCF method as carrier fields for the speech payload. All the other information in the RTP/UDP/IPv6 headers is needed to convey the speech payload in different environments and scenarios. The Checksum and the Source Port fields are part of every mini-packet (RTP + UDP + speech payload) within the MPkt. Therefore, 4-byte of the speech payload of every mini-packet will be truncated. Figure 2 shows the minipacket of the PMCF method.

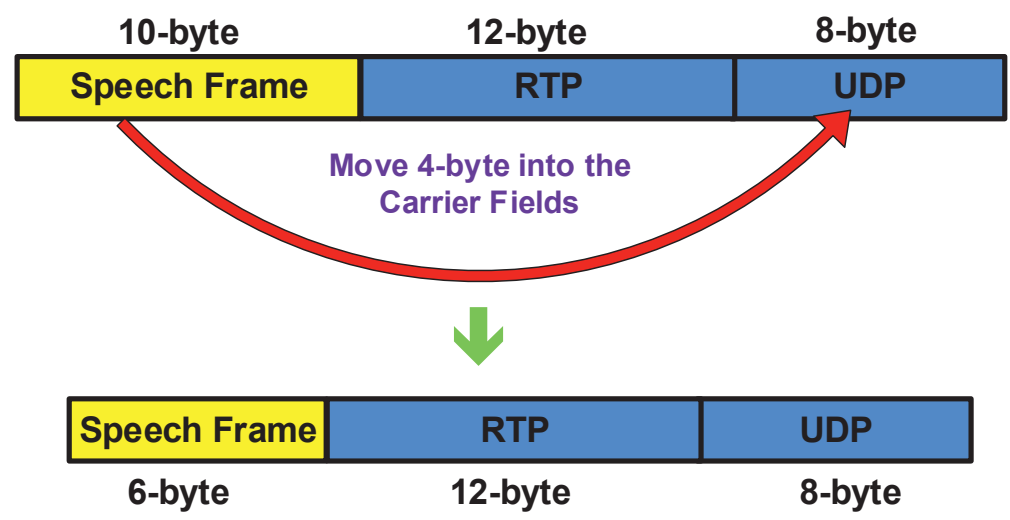

Figure 2. Mini-packet format

\subsection{The PMCFS Mechanism}

The PMCFS mechanism piggybacked several NPkt packets in one sizeable MPkt packet at the sender side. For each NPkt packet in the waiting queue, the mini-packet is extracted. Then, 4-byte of the speech payload of every mini-packet is placed in the Checksum and the Source Port fields of the minipacket header (RTP + UDP). This produces mini-packets with a smaller speech payload. After that, the produced mini-packets destined to the same destination will be piggybacked in one IPv6 header. Figure 3 shows the format of the MPkt packet. When the size reaches the MTU, or the waiting queue is empty of the VoIP packets, then the MPkt packet is transmitted to the destination. The destination IPv6 address of each call is stored in a particular table on both sender and receiver sides. This table is called the destination IPv6 address (Dst IPv6) table. The purpose of the Dst IPv6 table (Table3) is discussed in Subsection 3.4. Figure 4 shows the flowchart of the PMCFS mechanism.

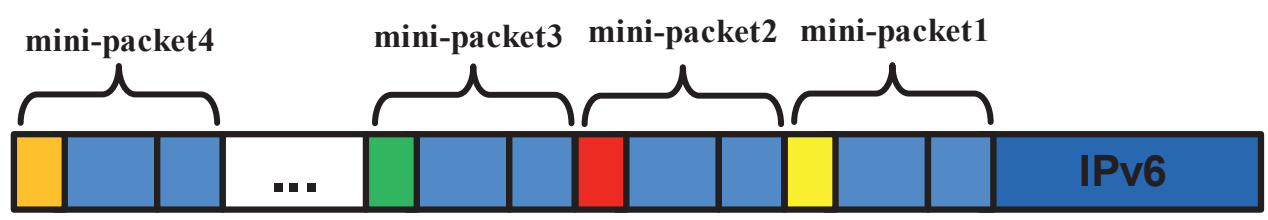

Figure 3. MPkt packet format 


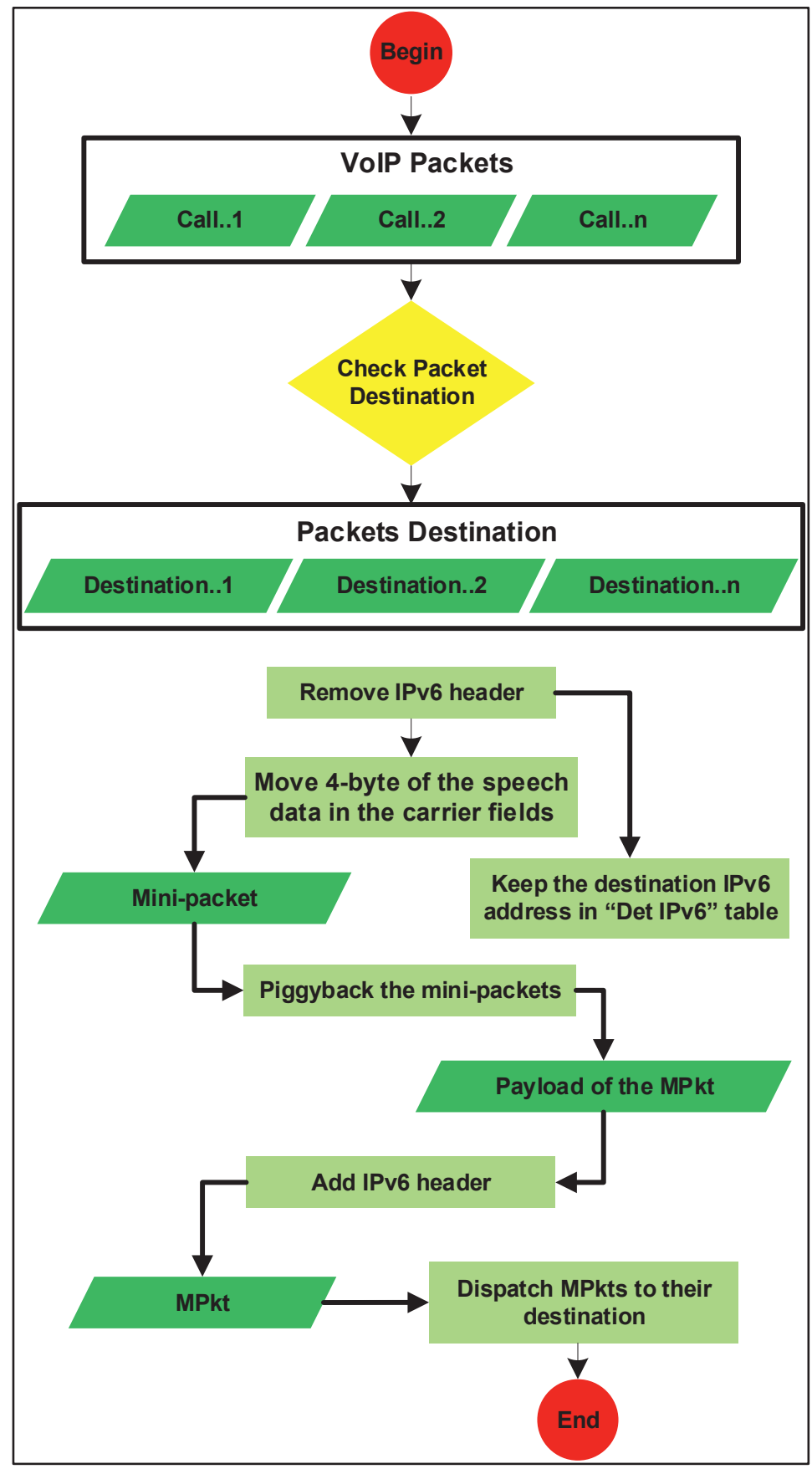

Figure 4. The PMCFS mechanism

\subsection{The PMCFR Mechanism}

The PMCFR mechanism segregates the MPkt packet to restore the original NPkt packets. At first, the IPv6 header is stripped out from the MPkt packet. Then, the mini-packets within the MPkt payload are separated. This is done based on the Length field in the UDP header of each mini-packet. Next, the speech data inside the Checksum and the Source Port fields are retrieved and placed at the end of the speech payload of the mini-packet. This constructs the mini-packet with the original full-length speech payload. Both Checksum and the Source Port fields are disabled to avoid misinterpreting them by the callee client. After that, the IPv6 header is attached to each mini-packet to construct the original NPkt packet. The destination IPv6 address of each NPkt packet is restored from the Dst IPv6 table (Table 3), as discussed in Subsection 3.4. Finally, the NPkt packet is sent to the callee client. Figure 5 shows the flowchart of the PMCFR mechanism. 


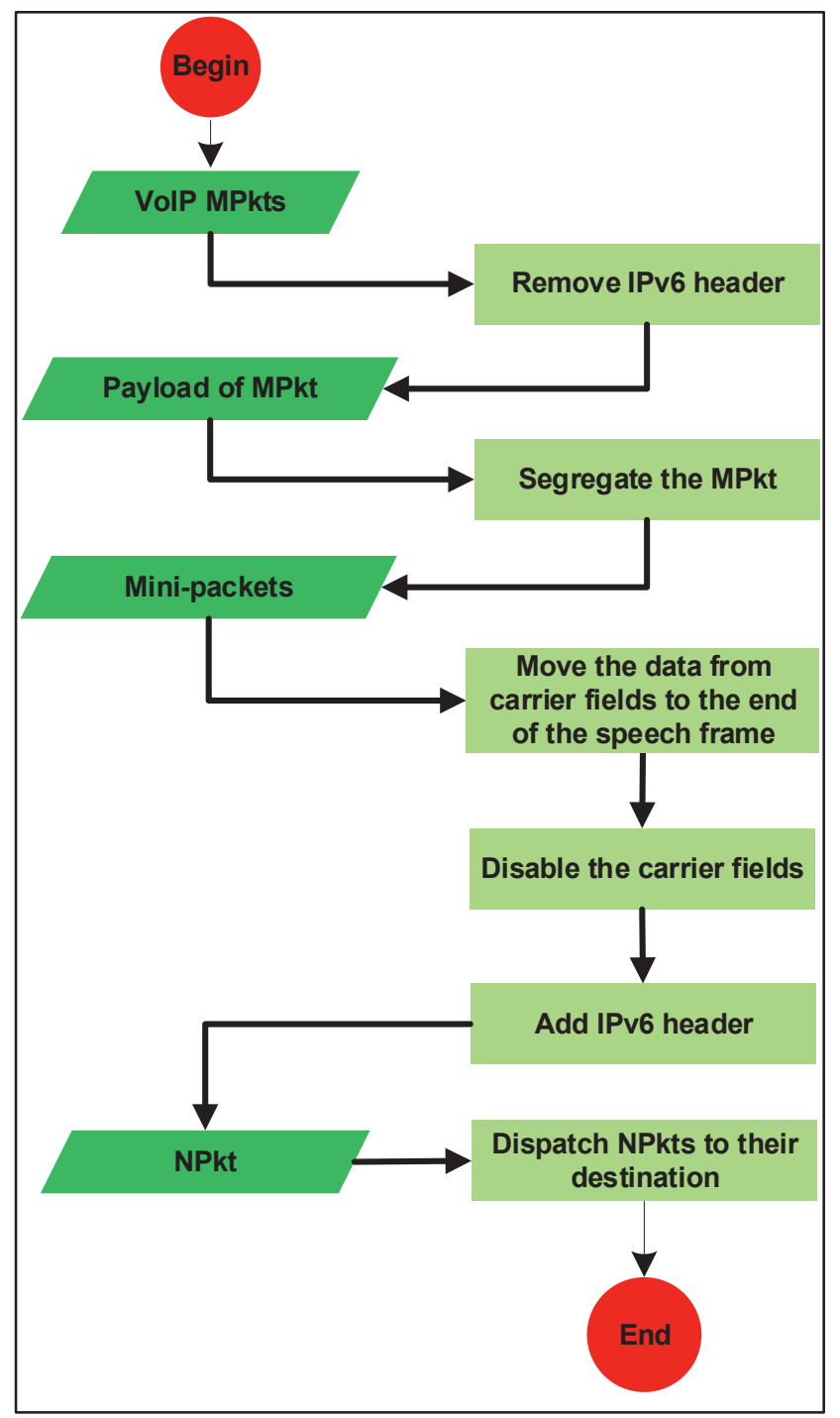

Figure 5. The PMCFR mechanism

\subsection{The Dst IPv6 Table}

The PMCFR mechanism adds the IPv6 header to the mini-packet, including the destination IPv6 address to construct the NPkt packet. The PMCF method keeps the destination IPv6 address in the Dst IPv6 table at both sender and receiver sides. A flag is needed to identify the destination IPv6 address of a specific call's packets. The PMCFR mechanism uses the SSRC in the RTP header for this purpose. The SSRC is a unique value in the RTP assigned for each call (Abualhaj et al., 2020b; Perkins, 2003). The SSRC is stored in the Dst IPv6 table along with its corresponding destination IPv6 address. When segregating the MPkt packet, the SSRC in the RTP of the mini-packet header is used to find the corresponding destination IPv6 address from the Dst IPv6 table. In sporadic cases, the SSRC of two calls could be matched. In these cases, the next available SSRC value is used. Table 3 exhibits the Dst IPv6 table at PMCFR and PMCFR.

Table 3. Dst IPv6 Table

\begin{tabular}{|l|l|l|l|l|l|}
\hline PACFS & & PACHR \\
\hline SSRC in RTP & SSRC in Dst IPv6 Table & Destination IPv6 & & SSRC & Destination IPv6 \\
\hline 126593730 & 126593730 & $2001: 1234:: 1 \mathrm{a} 13$ & & 126593730 & $2001: 1234:: 1 \mathrm{a} 13$ \\
\hline 126593731 & 126593731 & $2001: 1234:: 1 \mathrm{a} 14$ & & 126593731 & $2001: 1234:: 1 \mathrm{a} 14$ \\
\hline 126595730 & 126595730 & $2001: 1234:: 1 \mathrm{a} 15$ & & 126595730 & $2001: 1234:: 1 \mathrm{a} 15$ \\
\hline 126595730 & $126595731 *$ & $2001: 1234:: 1 \mathrm{a} 16$ & & 126595731 & $2001: 1234:: 1 \mathrm{a} 16$ \\
\hline 126593730 & $126593732 *$ & $2001: 1234:: 1 \mathrm{a} 17$ & 126593732 & $2001: 1234:: 1 \mathrm{a} 17$ \\
\hline$*$ Next available SSRC value
\end{tabular}




\section{PMCF Investigation}

This section investigates the impact of the PMCF on BWU when running VoIP over IPv6 networks. The PMCF was investigated compared to the typical IPv6 method (without multiplexing or carrier fields) and Roay method (Roay, 2013). The BWU improvement of the three methods was investigated in terms of call capacity, header size, bandwidth saving, and speech frame shortening metrics. The suggested PMCF technique along with the comparable methods were simulated on the wellknown Network Simulation 3 (NS3). The simulation topology includes two components of the PMCF technique, PMCFS and PMCFR, which discussed in sections 3. The simulation scenario assumes the PMCFS at the network exit-router at the sender side and the PMCFR at the network enter-router at the receiver side. The link between the two routers is represented as a buffer of 100 packets. Ten different experiments with different link bandwidths were used to compare the three methods using the metrics above. The link bandwidth started at $100 \mathrm{~kb}$ and increased by $100 \mathrm{~kb}$ for each different experiment up to $1000 \mathrm{~kb}$. Each of these experiments was run with two codecs, namely G.728 and G.726. A CBR (constant bit rate) traffic generator is used with each end.

\subsection{Calls Capacity}

Figures 6 and 7 display the call capacity of the PMCF method compared to the IPv6 method and Roay method, using G.26 and G.28 codecs, respectively. The PMCF method showed the highest call capacity with the two codecs compared to the IPv6 method and Roay method. In addition, the call capacity with G.28 codec has been promoted better than G.26 codec. For instance, the number of calls with the G.28 codec at $1000 \mathrm{~kb}$ bandwidth is 94, 81, and 35 when running the PMCF method, Roay method, and IPv6 method, respectively. Therefore, the call capacity is promoted by up to $269 \%$ and $231 \%$ over the typical IPv6 method, when using the PMCF method and Roay method, respectively.

On the other hand, the number of calls with the G.26 codec at $1000 \mathrm{~kb}$ bandwidth is 68,61 , and 31 when running the PMCF method, Roay method, and IPv6 method, respectively. Therefore, the call capacity is promoted by up to $219 \%$ and $197 \%$ over the typical IPv6 method when using the PMCF method and Roay method, respectively. The apparent reason for the call's capacity promotion is piggybacking several NPkt packets in one MPkt packet with one IPv6 header rather than a dedicated IPv6 header to each NPkt. However, the G.28 codec shows higher call capacity promotion than G.26 codec because the speech payload of the G.26 codec is 20-bytes while the speech payload of the G.28 codec is 10-bytes. Thus, placing 4-byte of speech payload in the carrier fields will impact the 10-byte G.28 codec more than the 20-byte G.26 codec. Therefore, the smaller the speech payload of a codec, the higher the call capacity is promoted when using the PMCF method.

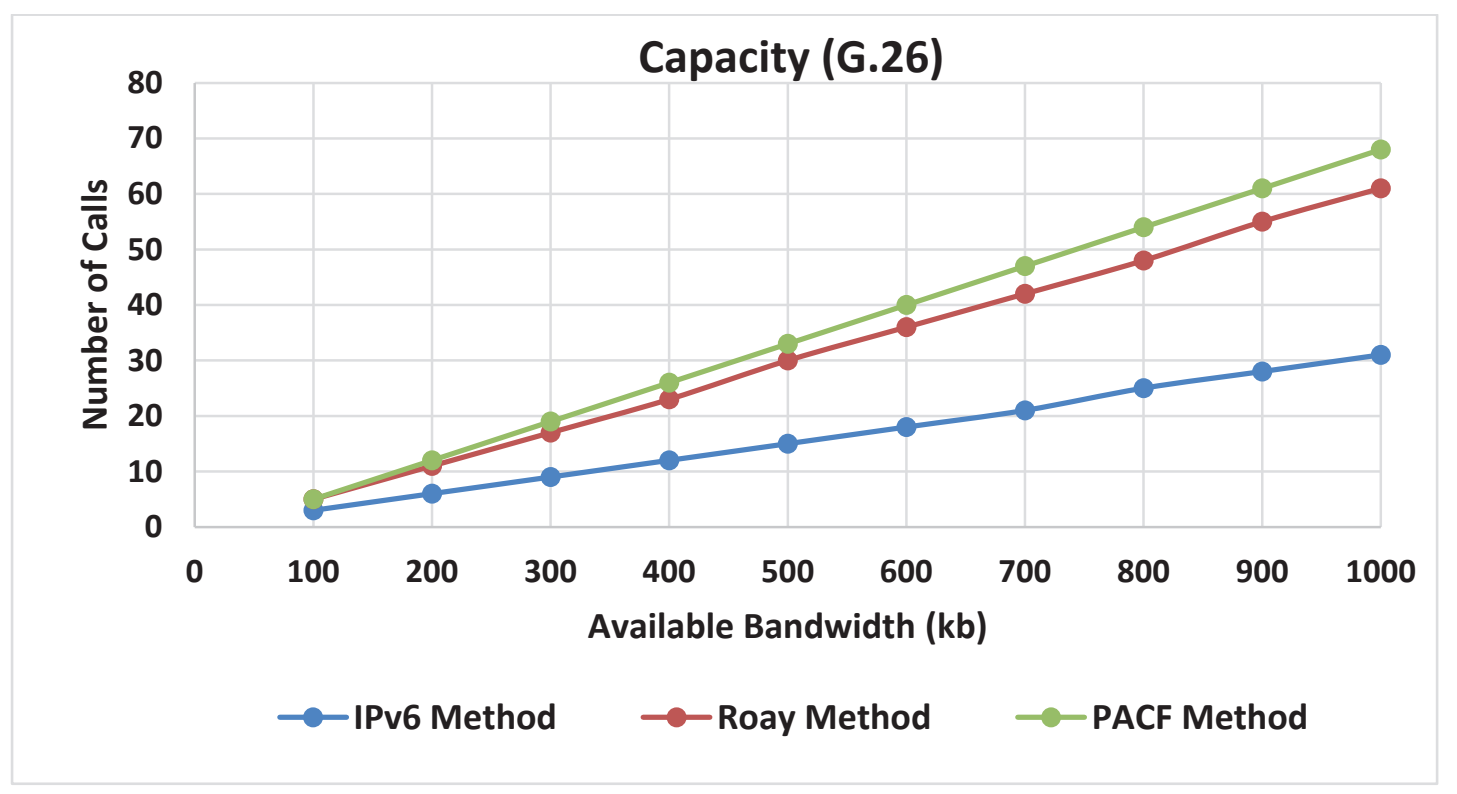

Figure 6. Call capacity (G.26 codec) 


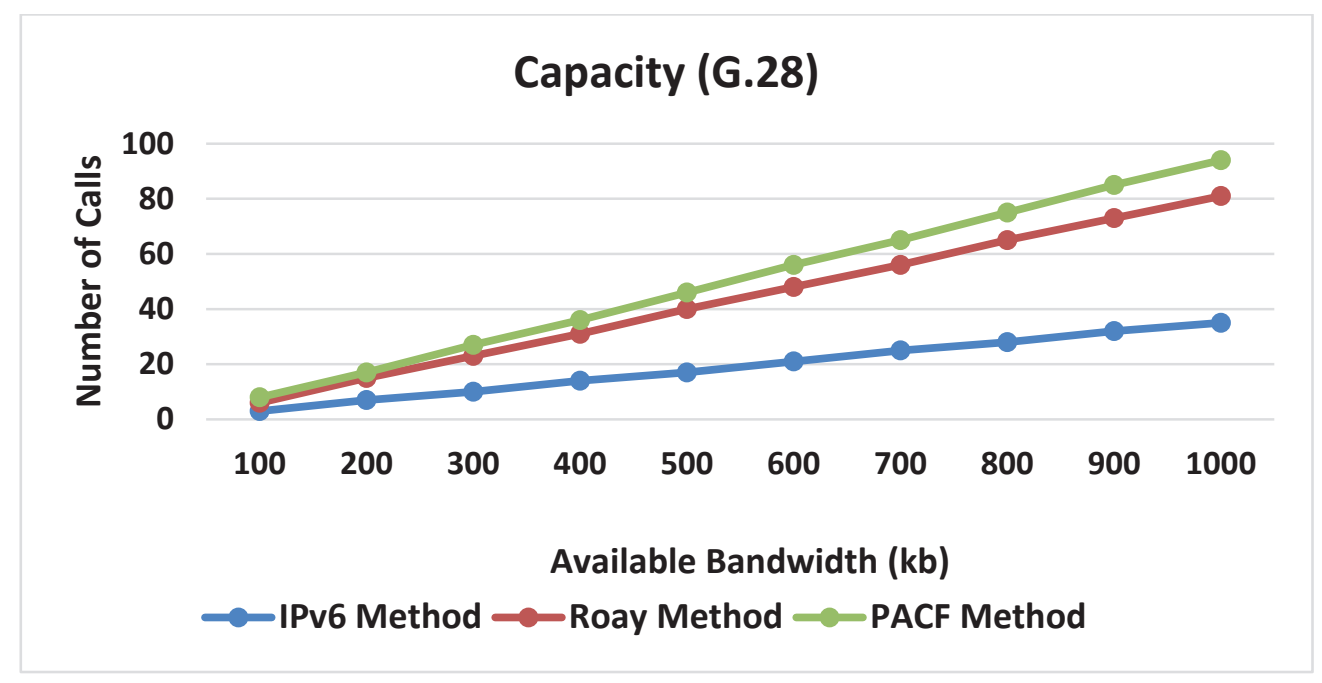

Figure 7. Call capacity (G.28 codec)

\subsection{Header Size}

Figure 8 displays the header size ratio of the PMCF method compared to the IPv6 method and Roay method using G.26 codec. The PMCF method showed the lowest header size ratio compared to the IPv6 method and Roay method. For instance, the header size ratio at 100 calls is $45 \%, 51 \%$, and $75 \%$ when running the PMCF method, Roay method, and IPv6 method, respectively. Therefore, the wasted bandwidth when running the PMCF method is less than the other comparable methods. This is due to piggybacking several NPkt packets in one MPkt packet with one IPv6 header rather than a dedicated IPv6 header to each NPkt. In addition, the 4-byte Checksum and the Source Port carrier fields are not considered header fields because they are carrying the part of the speech samples.

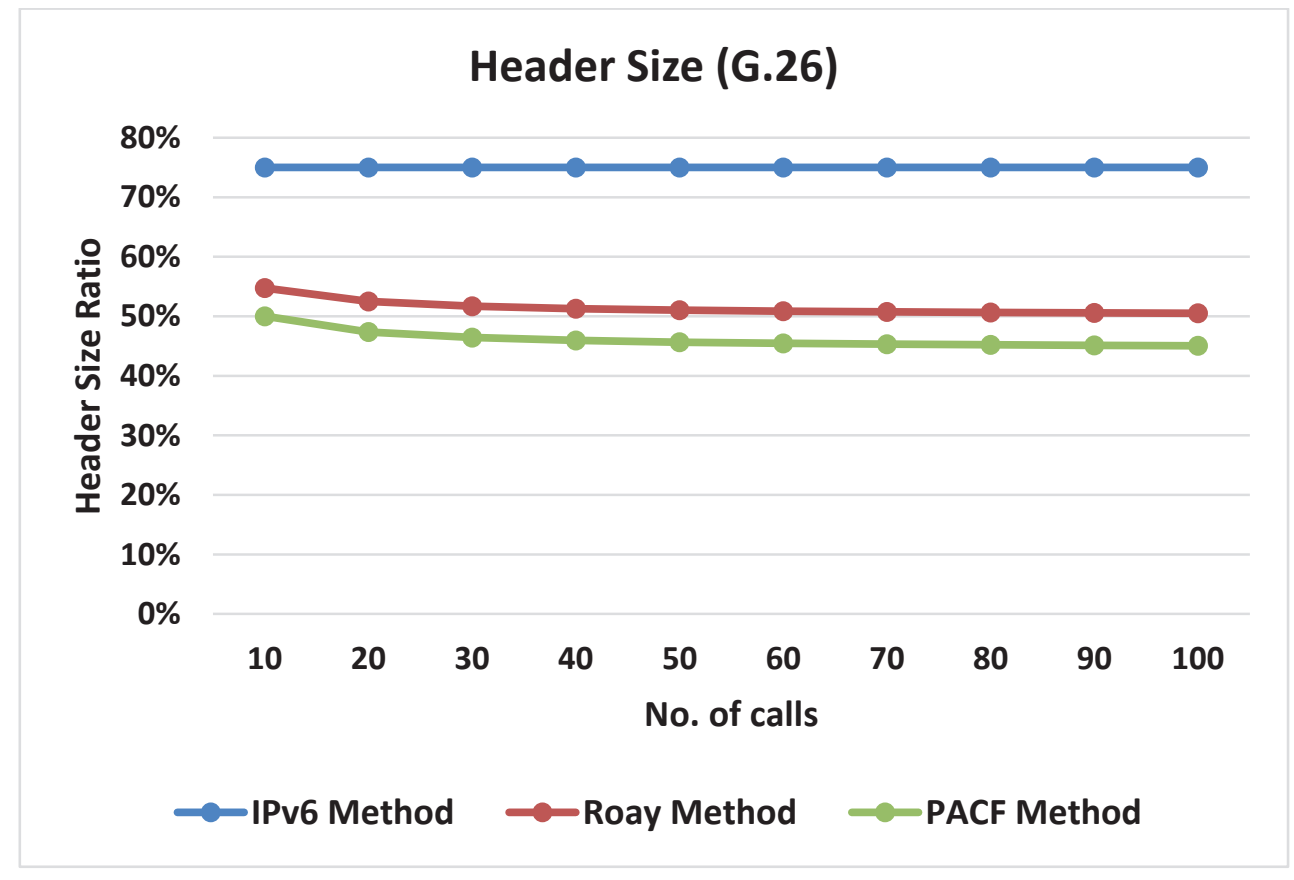

Figure 8. Header size ratio

\subsection{Bandwidth Saving}

Figures 9 and 10 display the bandwidth saving of the PMCF method and Roay method compared to the traditional IPv6 method, using G.26 and G.28 codecs, respectively. Again, with the two codecs, the 
PMCF method showed higher bandwidth saving than Roay method. In addition, the bandwidth saving with G.28 codec has promoted better than G.26 codec. For instance, the bandwidth saving with the G.28 codec at 100 calls is $62 \%$ and $57 \%$ when running the PMCF method and Roay method compared to the IPv6 method, respectively. Therefore, the PMCF method showed better bandwidth saving by up to 5\% than the Roay method. On the other hand, the PMCF method showed bandwidth saving with the G.26 codec equals $55 \%$ at 100 calls which is less than the G.28 codec. The apparent reasons for the bandwidth saving are the exact reasons for promoting the capacity of the call.

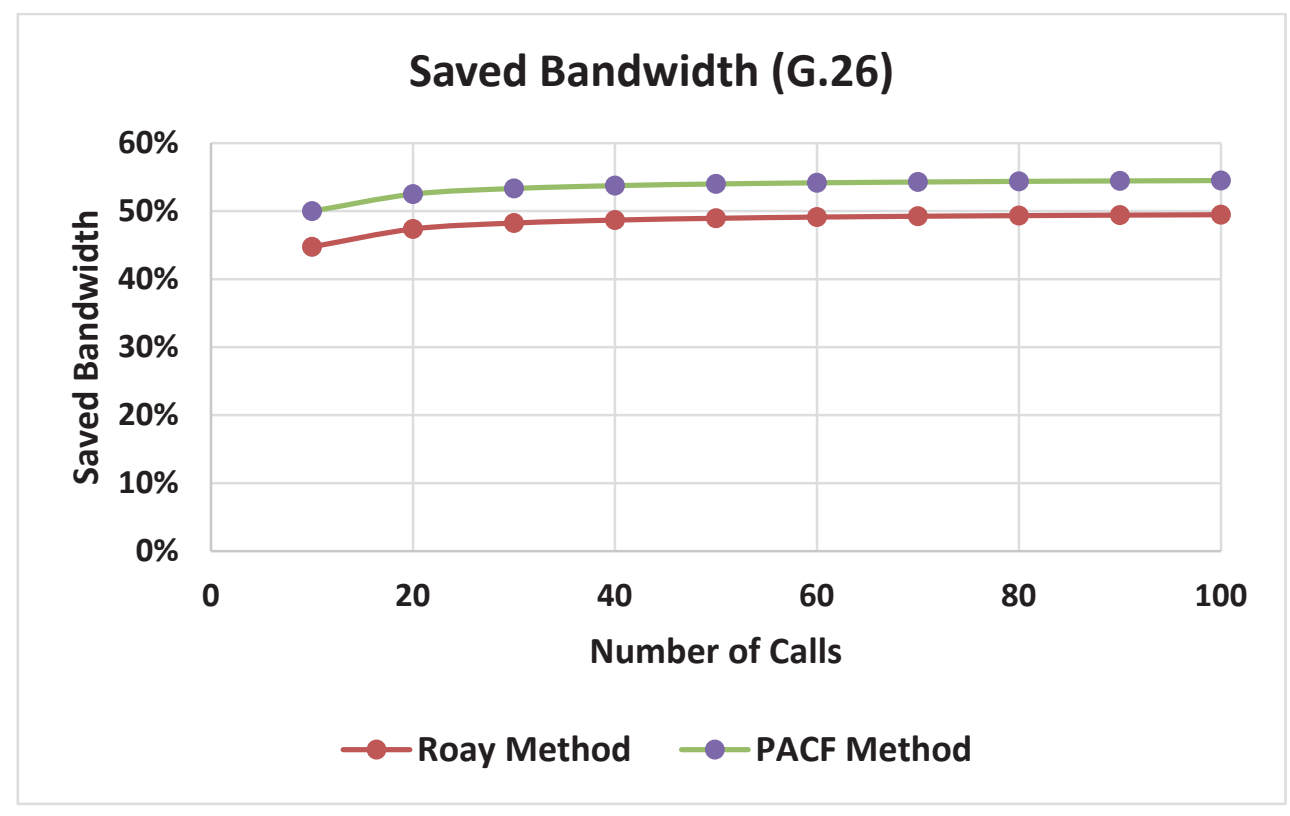

Figure 9. Bandwidth saving (G.26 codec)

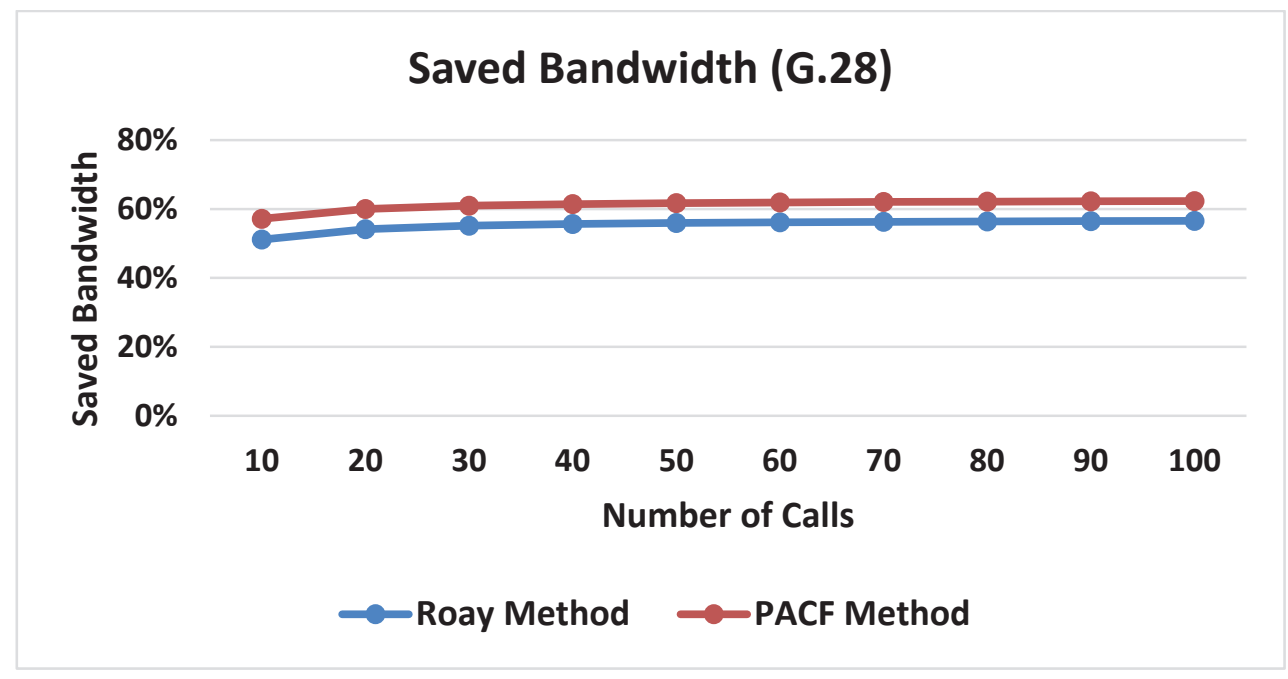

Figure 10. Bandwidth saving (G.28 codec)

\subsection{Speech Frame Shortening}

Figure 11 displays the speech frame shortening ratio when using the PMCF method. The shortening ratio is $20 \%$ and $40 \%$ when using the G.26 codec and G. 28 codec, respectively. The resulting shortening is due to place part of the speech frame in the 4-byte carrier fields. In addition, the shorting ratio is more when using the G.28 codec compared to the G.26 codec. This is because the speech frame of the G.28 codec is smaller than the speech frame of the G.26 codec. Therefore, the smaller the speech payload of a codec, the higher the shortening ratio. 


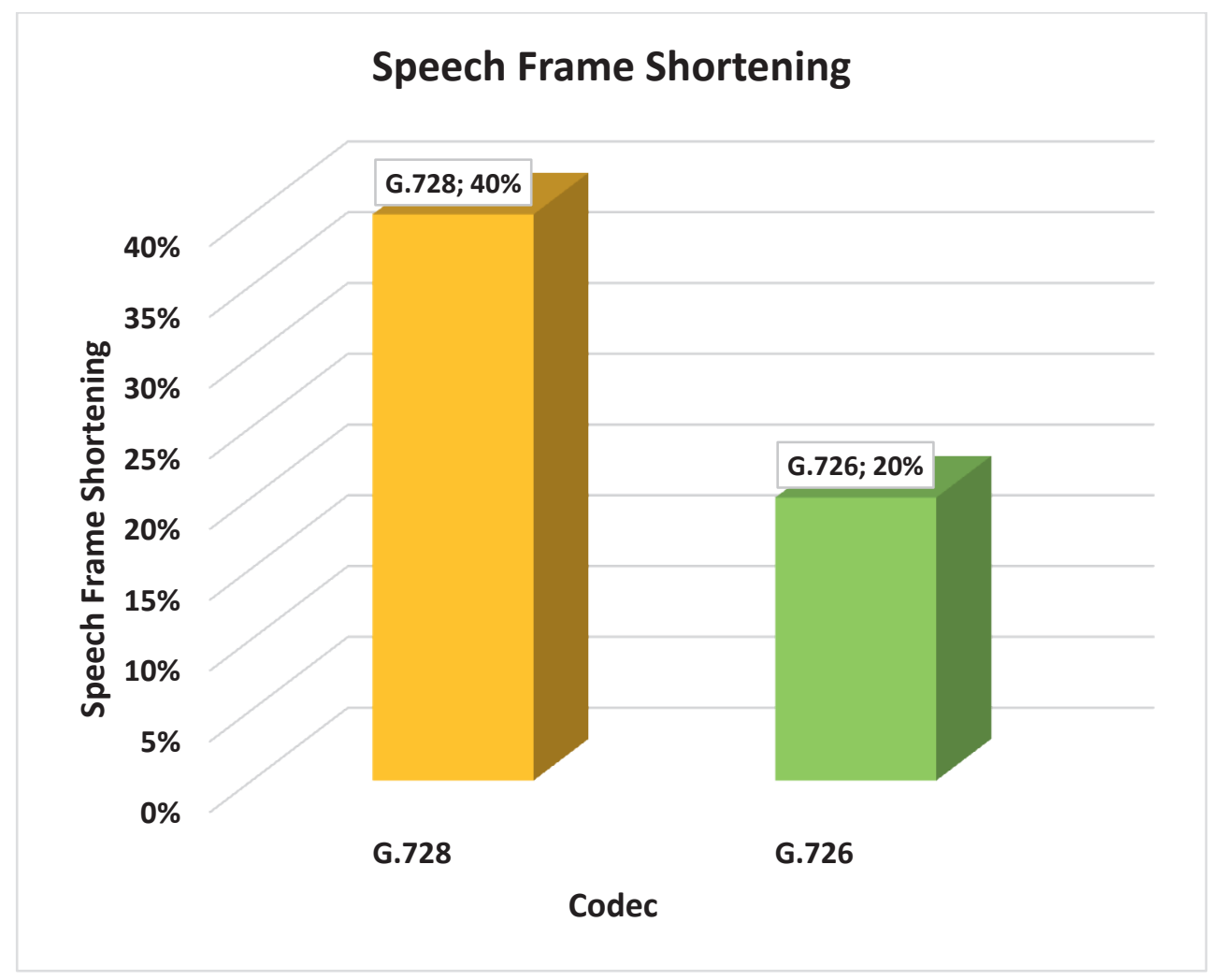

Figure 11. Speech frame shortening ratio

\section{Conclusion}

VoIP has played a crucial role in virtual communication during the COVID-19 pandemic lockdown. Unfortunately, the size of the VoIP packet header, particularly over IPv6 networks, is consuming most of the bandwidth, with little bandwidth remaining for the actual speech data. The paper has proposed the PCMF method to promote the BWU of VoIP over IPv6 networks. The PCMF method piggybacked many IPv6 VoIP packets in one IPv6 header. It also uses the Checksum and Source Port fields in the UDP header of each mini-packet to carry a portion of the speech frame. These two techniques have promoted the BWU considerably. For instance, the call capacity has been boosted by up to $269 \%$ when using the G.28 codec at 1000kb network bandwidth. In addition, the bandwidth saving with the same G.28 codec at 100 calls is $62 \%$ of the available bandwidth compared to the IPv6 method. This promotion of the BWU will also boost the quality of the calls. Therefore, the PMCF method can work successfully to promote VoIP's overall performance over IPv6 networks. As future works, the impact of the PCMF method on the VoIP security will be investigated.

\section{References}

1. Abualhaj, M. M., Al-Tahrawi M. M. and Al-Zyoud, M. (2021a) Contracting VoIP Packet Payload Down to Zero. CYBERNETICS AND INFORMATION TECHNOLOGIES, 21(1). DOI:10.2478/cait2021-0010.

2. Abualhaj, M. M., Shambour, Q. Y., Hussein, A. H. and Kharma, Q. M. (2021b). Down to Zero Size of VoIP Packet Payload. CMC-COMPUTERS MATERIALS \& CONTINUA, 68(1), 1271-1283. DOI:10.32604/cmc.2021.014928.

3. Abualhaj, M. M., et al. (2020a) Survey and analysis of VoIP frame aggregation methods over AMSDU IEEE 802.11 n wireless networks. Comput. Mater. Contin, 66, 1283-1300. DOI:10.32604/cmc.2020.012991.

4. Abualhaj, M. M., Sambour, Y. Q. and Hussein H. A. (2020b) Effective packet multiplexing method to improve bandwidth utilisation. International Journal of Computer Applications in Technology, 63(4), 327-336. DOI: 10.1504/IJCAT.2020.110410. 
5. Abu-Alhaj, M. M., Kolhar, M. S., Chandra, L. V., Abouabdalla, O. and Manasrah, A. M. (2010) Delta-multiplexing: A novel technique to improve VoIP bandwidth utilization between VoIP gateways. In: 2010 10th IEEE International Conference on Computer and Information Technology, Bradford, UK, IEEE, 329-335. DOI: 10.1109/CIT.2010.86.

6. Casner, S. and Jacobson, V. (1999) Compressing IP/UDP/RTP Headers for Low-Speed Serial Links, RFC 2508. DOI:https://doi.org/10.17487/RFC2508.

7. Eggert L., Fairhurst G. and Shepherd G. (2017) UDP usage guidelines, IETF RFC 8085. DOI: $10.17487 / \mathrm{rfc} 8085$.

8. Gao, J., Li, Y., Jiang, H., Liu, L. and Zhang, X. (2019) An RTP Extension for Reliable User-Data Transmission over VoIP Traffic. In: International Symposium on Security and Privacy in Social Networks and Big Data, Singapore, Springer, 74-86. DOI:10.1007/978-981-15-0758-8_6.

9. Garro-Abarca, V., Palos-Sanchez, P. and Aguayo-Camacho, M. (2021) Virtual Teams in Times of Pandemic: Factors That Influence Performance. Frontiers in Psychology, 12, 232. DOI:10.3389/fpsyg.2021.624637.

10. Gupta, N., Kumar, N. and Kumar, H. (2018) Comparative analysis of voice codecs over different environment scenarios in VoIP. In: 2018 Second International Conference on Intelligent Computing and Control Systems (ICICCS), India, Madurai, IEEE, 540-544. DOI: 10.1109/ICCONS.2018.8663241.

11. Hartpence, B. (2013) Packet Guide to Voice over IP: A system administrator's guide to VoIP technologies. O'Reilly Media, Inc.

12. Hoffman, J. (2019). VOIP Adoption Statistics for 2019 \& Beyond. Available on the internet: [https://wisdomplexus.com/blogs/voip-adoption-statistics-2019-beyond/]

13. Khan, S. A., Moosa, M., Naeem, F., Alizai, M. H. and Kim, J. M. (2016) Protocols and mechanisms to recover failed packets in wireless networks: History and evolution. IEEE Access, 4, 4207-4224. DOI: 10.1109/ACCESS.2016.2593605.

14. Narayan, S., Gordon, M., Branks, C. and Fan, L. (2010) VoIP network performance evaluation of operating systems with IPv4 and IPv6 network implementations. In: 2010 3rd International Conference on Computer Science and Information Technology, China, Chengdu, IEEE, 5, 669-673. DOI: 10.1109/ICCSIT.2010.5564004.

15. Perkins, C. (2003) RTP: Audio and Video for the Internet. Addison-Wesley Professional.

16. Raycheva, L., Velinova, N., Miteva, N. and Tomov, M. (2020) Impacts of Virtual Communication During Social Isolation of Covid'19. In: International Conference on Human Systems Engineering and Design: Future Trends and Applications, Cham, Springer, 63-68. DOI:10.1007/978-3-03058282-1_11.

17. Roay, B. (2013) Generic UDP multiplexing for voice over internet protocol (VOIP). U.S. Patent No. $8,553,692$.

18. Seytnazarov, S. and Kim, Y. T. (2017) QoS-aware adaptive A-MPDU aggregation scheduler for voice traffic in aggregation-enabled high throughput WLANs. IEEE Transactions on Mobile Computing, 16(10), 2862-2875. DOI:10.1109/CNSM.2014.7014193.

19. Stein, S. and Rippel, E. (2020) Efficient double parity forward error correction on a communication network, U.S. Patent No. 10,567,102.

20. Subbiah, B., Sengodan, S. and Rajahalme, J. (1999) RTP payload multiplexing between IP telephony gateways. In: Seamless Interconnection for Universal Services. Global Telecommunications Conference. GLOBECOM'99. (Cat. No. 99CH37042), IEEE, 2, 1121-1127. DOI: 10.1109/GLOCOM.1999.829948.

21. Sze, H. P., Liew, S. C., Lee, J. Y. and Yip, D. C. (2002). A multiplexing scheme for H. 323 voiceover-IP applications. IEEE Journal on Selected Areas in Communications, 20(7), 1360-1368.

22. Tomoskozi, M., Seeling, P., Ekler, P. and Fitzek, F. H. (2016) Regression model building and efficiency prediction of rohcv2 compressor implementations for voip. In: 2016 IEEE Global Communications Conference (GLOBECOM), Washington, DC USA, IEEE, 1-6. DOI: 10.1109/GLOCOM.2016.7842207.

23. Tomsho, G. (2012) Guide to networking essentials. Cengage Learning. 